\title{
First Signal on the Cryogenic Fourier-Transform Ion Cyclotron Resonance Mass Spectrometer
}

\author{
Cheng Lin,, ${ }^{*}, \mathrm{~b}$ Raman Mathur, ${ }^{*, a, c}$ Kostantin Aizikov, ${ }^{\mathrm{a}, \mathrm{c}}$ \\ and Peter B. O'Connor ${ }^{\mathrm{a}, \mathrm{b}, \mathrm{c}}$ \\ ${ }^{a}$ Cardiovascular Proteomics Center, Boston University School of Medicine, Boston, Massachusetts, USA \\ ${ }^{\mathrm{b}}$ Mass Spectrometry Resource, Department of Biochemistry, Boston University School of Medicine, Boston, \\ Massachusetts, USA \\ ${ }^{\mathrm{c}}$ Department of Electrical and Computer Engineering, Boston University, Boston, Massachusetts, USA
}

\begin{abstract}
The construction and achievement of the first signal on a cryogenic Fourier-transform ion cyclotron resonance mass spectrometer (FTICR-MS) are reported here, demonstrating proof-of-concept of this new instrument design. Building the FTICR cell into the cold bore of a superconducting magnet provided advantages over conventional warm bore design. At $4.2 \mathrm{~K}$, the vacuum system cryopumps itself, thus removing the requirement for a large bore to achieve the desired pumping speed for maintaining base pressure. Furthermore, because the bore diameter has been reduced, the amount of magnet wire needed to achieve high field and homogeneity was also reduced, greatly decreasing the cost/Tesla of the magnet. The current instrument implements an actively shielded 14-Tesla magnet of vertical design with an external matrix-assisted laser desorption/ ionization (MALDI) source. The first signal was obtained by detecting the laser desorbed/ionized (LDI) $\mathrm{C}_{60}^{+\cdot}$ ions, with the magnet at 7 Tesla, unshimmed, and the preamplifier mounted outside of the vacuum chamber at room temperature. A subsequent experiment done with the magnet at 14 Tesla and properly shimmed produced a $C_{60}$ spectrum showing approximately 350,000 resolving power at $m / z \sim 720$. Increased magnetic field strength improves many FTMS performance parameters simultaneously, particularly mass resolving power and accuracy. (J Am Soc Mass Spectrom 2007, 18, 2090-2093) (C) 2007 American Society for Mass Spectrometry
\end{abstract}

$\mathrm{R}$ ecent advances in Fourier-transform ion cyclotron resonance mass spectrometry (FTICR-MS, or FTMS) [1] have made it possible to routinely generate mass spectra with sub part per million (ppm) mass accuracy [2-5] and ultrahigh resolving power (with RP >8,000,000 reported at $8.6 \mathrm{kDa}$ ) [6]. Mass accuracy and resolving power are critical and determining performance parameters for top-down [7] and bottom-up $[8,9]$ proteomics experiments, as well as petroleomics [10]. Many performance parameters of FTMS, particularly mass resolving power and accuracy, increase linearly with the magnetic field strength [11]. Currently, the highest field used in commercially available FTMS instruments is $15 \mathrm{~T}$. Although nuclear magnetic resonance (NMR) magnets with up to $21 \mathrm{~T}$ magnetic field are commercially available, they are rarely used in FTMS instruments because the narrow bore diameter limits the obtainable pumping speed and base pressure. Increasing the bore diameter, however, increases the

Address reprint requests to Dr. Peter B. O'Connor, Boston University School of Medicine, Mass Spectrometry Resource, Department of Biochemistry, 670 Albany St., Boston, MA 02118, USA. E-mail: poconnor@bu.edu

* These authors contributed equally to this work. cost of the magnet (roughly quadratically) and greatly increases the difficulty of constructing a stable high homogeneity magnet. As a result, modern FTMS instruments have to make a compromise on bore diameter to ensure enough pumping speed to maintain the desirable base pressure, which limits the high field magnets currently accessible. In 2002, a new design, called the cryogenic FTMS, was proposed in which the FTMS would be constructed inside the $4.2 \mathrm{~K}$ cold bore of a superconducting magnet [12]. At $4.2 \mathrm{~K}$, instead of outgassing, the surfaces of the vacuum system become cryopumping, making it possible to achieve sufficient pumping speed $\left(>10^{5} \mathrm{~L} / \mathrm{s}[13]\right)$ and low base pressure $\left(<1 \times 10^{-16}\right.$ mbar [14]) with a narrow bore magnet. Moreover, the insulation between the cold bore that houses the magnet and the warm bore within which the FTMS is constructed can be removed in the cryogenic FTMS design, further increasing the available bore diameter. Finally, in addition to the gain in magnetic field strength, pumping speed, and base pressure, the cryogenic FTMS offers a third advantage in that the Johnson noise in the preamplifier's input resistor can be reduced by a factor of 8.4 by cooling the preamplifier to $4.2 \mathrm{~K}$. 
There are considerable, but manageable, challenges associated with the construction and operation of such a cryogenic FTMS that must be overcome. Particularly, the helium boiloff will greatly increase due to the increased heat transfer into the Dewar with the immersion of a metal chamber into the cryogenic magnet bore. This communication presents the construction of a cryogenic FTMS following closely the design proposed in the previous paper to solve these difficulties, and shows the first signal achieved on this instrument.

\section{Experimental}

\section{Magnet Design}

The cutaway view of the complete cryogenic FTMS with its salient components is shown in Figure 1. It incorporates an actively shielded 15-Tesla superconducting magnet (Cryomagnetics, Oak Ridge, TN, USA) with a vertical, cryogenic temperature bore, and homogeneity of about $12 \mathrm{ppm}$ over a $5 \times 5 \mathrm{~cm}$ cylindrical region. The system is designed to have zero loss in liquid helium under normal operation, with the equipped Sumitomo RDK415 cryo-refrigerator (Sumitomo Heavy Industries, Tokyo, Japan) providing a cooling power of about $80 \mathrm{~W}$ at $77 \mathrm{~K}$ and about $1.5 \mathrm{~W}$ at $4.2 \mathrm{~K}$ to compensate for the heat transfer into the magnet Dewar. The Dewar can hold up to $100 \mathrm{~L}$ of liquid helium, which facilitates initial cool down, and provides a reservoir of cryogen to prevent magnet quenching in case of power outage and/or unaccounted heat loads. Two

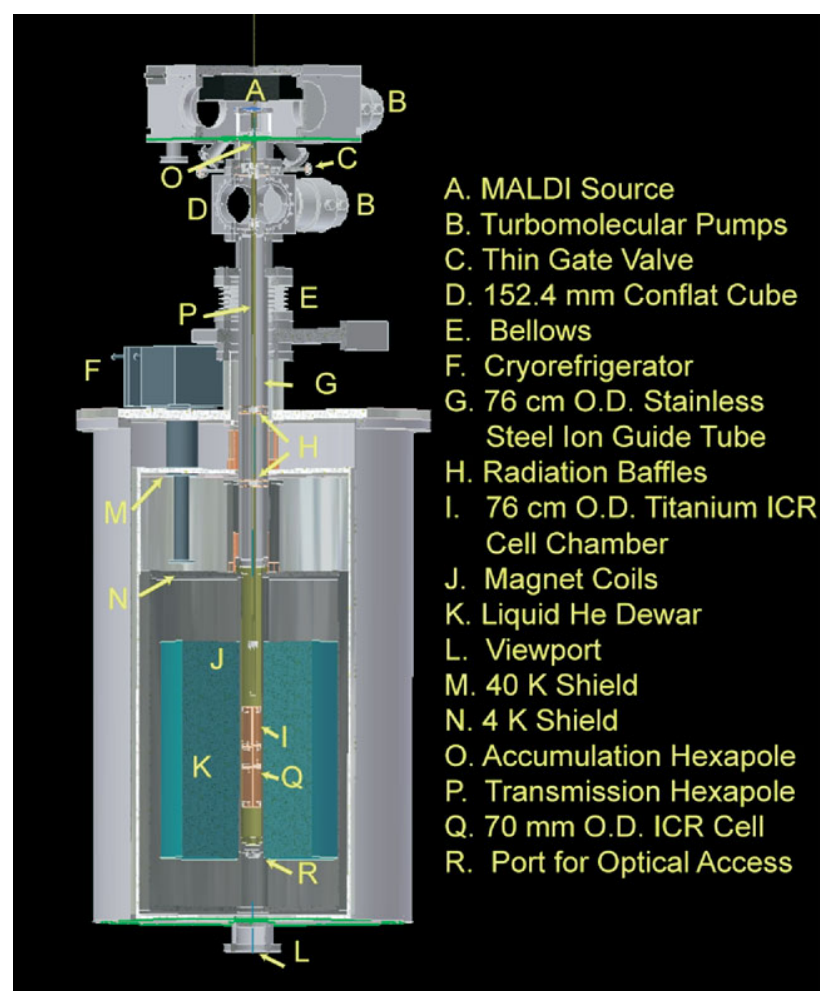

Figure 1. Cutaway view of the complete cryogenic FTMS. radiation-shielded ultrahigh vacuum (UHV) chambers separate the outer surrounding environment from the magnet chamber, which is also radiation shielded from the $4 \mathrm{~K}$ inner chamber housing the FTMS, allowing for short-duration temperature shocks. There is a $3.4 \mathrm{~cm}$ conflat port at the bottom of the Dewar for optical access offering the capability of carrying out infrared multiphoton dissociation (IRMPD) experiments.

\section{FTMS}

The FTMS consists of an external matrix-assisted laser desorption/ionization (MALDI) source [15], multipole ion guides, and an open cylindrical ICR cell [16]. The high-pressure MALDI source is similar to the one described previously [17], where analytes are desorbed and ionized from the target plate mounted on an XY-stage (Fraunhofer USA, Brookline, MA, USA) by focused $355 \mathrm{~nm}$ light from a Nd:YAG laser (Continuum, Santa Clara, CA, USA). The ion transfer optics include an accumulation hexapole with front and back trapping plates that can trap ions externally, and a transmission hexapole, both driven by custom RF oscillators [18] but separated by a thin gate valve to maximize ion transmission [19]. The ICR cell has an outer diameter (o.d.) of $7 \mathrm{~cm}$, with $5 \mathrm{~cm}$ long central excite/detect electrodes, a $10 \mathrm{~cm}$ long inner trapping ring on each side to extend the trapping volume, and two outer trapping plates. A $120 \mathrm{~h}$ bakeout resulted in a vacuum at the cube of about $1.5 \times 10^{-9} \mathrm{mbar}$. This cube is about $130 \mathrm{~cm}$ from the ICR cell, where the base pressure is expected to be lower because of cryopumping [14]; this assumption must be tested with further experiments.

\section{Data Acquisition}

A PXI-based data system (National Instruments, Austin, TX, USA) developed at FOM-AMOLF [20] was used to control the pulse sequence, generate the excite waveform, and acquire the spectrum. The excite waveform was produced using an arbitrary waveform generator, followed by amplification and balancing through an ironcore transformer before transmission to the excite plates. The current setup uses an op-amp-based standard instrumentation amplifier at room temperature outside of the vacuum system to amplify the induced ICR signal, so the hypothesized noise reduction is not expected with these initial results. The digitized transients were zero-filled once and fast Fourier transformed without apodization to produce the magnitude mode mass spectra shown.

\section{Results and Discussion}

First Signal at 7 Tesla

$\mathrm{C}_{60}$ was chosen as the analyte for tuning the cryogenic FTMS instrument. To reduce the tuning space dimension, the LDI mass spectrum of $\mathrm{C}_{60}$ was first obtained and optimized on a room temperature $7 \mathrm{~T}$ MALDI- 
FTMS instrument [21]. The data system, excite, and detect amplifiers used in that experiment were then transported to the cryogenic FTMS, with its magnet also charged halfway to $7 \mathrm{~T}$ to allow direct usage of the previously optimized pulse sequence. With parameters such as ion time-of-flight, voltage settings on ion lenses, hexapole RF amplitudes and frequencies, and the excite amplitude and duration set near optimal values, acquiring the $\mathrm{C}_{60}$ spectrum in the cryo-FTMS was expected to require minimal tuning.

$\mathrm{C}_{60}$ molecules were first desorbed from a stainless steel plate and ionized by a single laser shot of around $80 \mu \mathrm{J}$ (focused to $\sim 100 \mu \mathrm{m}$ diameter spot) at $355 \mathrm{~nm}$, then transferred to the ICR cell through two hexapoles, both operated at $900 \mathrm{kHz}$ and about $25 \mathrm{~V}_{\mathrm{pp}}$ amplitude. Gated trapping was used to trap the ions in the ICR cell, with the lower inner trapping ring held at $10 \mathrm{~V}$, and the upper one briefly dropped to $-1 \mathrm{~V}$ to allow the passage of ions into the cell before returning to $10 \mathrm{~V}, 1.27 \mathrm{~ms}$ after the laser fired, corresponding to a $1.27 \mathrm{~ms}$ ion flight time. After a short delay of about $200 \mathrm{~ms}$, the trapped ions were subjected to a broadband chirp excitation $(m / z 500-1000 \mathrm{Da}, 107-214 \mathrm{kHz}$ at $7 \mathrm{~T})$ for 20 ms at $70 \%$ attenuation $\left(\sim 20 \mathrm{~V}_{\mathrm{pp}}\right)$, and the image current was detected at $4 \mathrm{MHz}$ sampling rate and with $1 \mathrm{M}$ point (14 bit) buffer size, corresponding to a $0.26 \mathrm{~s}$ transient. Figure 2 shows the optimized single scan mass spectrum of $\mathrm{C}_{60}^{+}$at $7 \mathrm{~T}$, with the inset showing the time domain signal, which clearly displays the beat pattern of the closely spaced isotopic peaks. The mass resolving power, $\mathrm{M} / \Delta \mathrm{M}_{\mathrm{FWHM}}$, is around 18,000 , close to the theoretical Fourier-transform limit $\left(f^{*} t / 2\right.$ [22] of 19,344).

\section{4-Tesla Spectrum}

Although the current magnet is designed for $15 \mathrm{~T}$, shimming values are available only at $14 \mathrm{~T}$. Thus, after optimizing the $\mathrm{C}_{60}^{+}$signal at $7 \mathrm{~T}$, the magnet was brought up to $14 \mathrm{~T}$, and shimmed for optimal overall homogeneity. Figure 3 shows a single-scan spectrum of $\mathrm{C}_{60}^{+}$at $14 \mathrm{~T}$ with broadband excitation and an $8.4 \mathrm{~s}$ transient. The mass resolving power is about 350,000 , falling far short of the theoretical limit of about

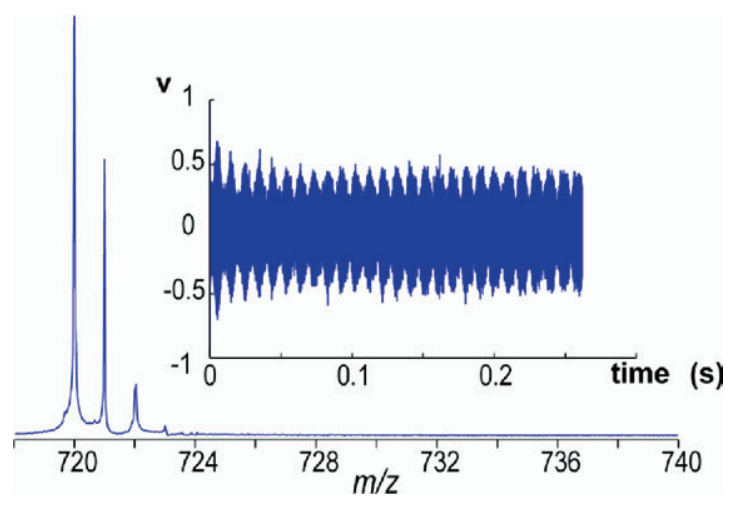

Figure 2. First signal on cryogenic FTMS at 7 Tesla.

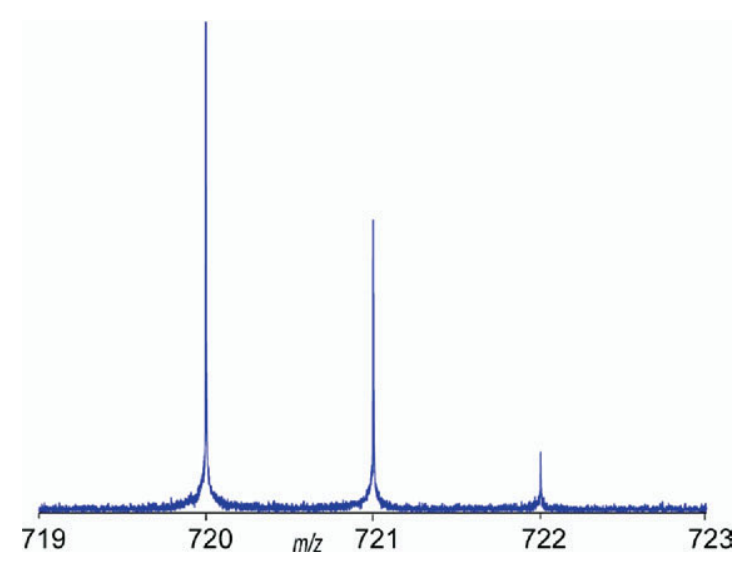

Figure 3. High-resolution mass spectra of $\mathrm{C}_{60}$ at 14 Tesla.

$1,250,000$. Such deviation likely results from dephasing of the ion clouds, particularly because the high trapping potentials used increases trapping field inhomogeneities and the magnetron expansion rate.

\section{Helium Boiloff}

Building the FTMS directly into the cold magnet bore will inevitably increase the heat load on the magnet Dewar, resulting in a faster helium boiloff rate. Although the use of a cryo-refrigerator partially compensates for the heat transfer into the magnet Dewar, it is also important to minimize heat transfer to minimize boiloff. Because the magnet chamber and the FTMS are both inside UHV enclosures, convective heat transfer is negligible. Radiative heat transfer is minimized by installing radiation baffles along the ion optical axis, and putting many layers of super-insulation materials on the surfaces of the radiation shields. Conductive heat transfer can be readily estimated by the heat transfer equation [23]:

$$
\dot{Q}_{\text {conductive }}=k(T) A \frac{\partial T}{\partial x}
$$

where $k(T)$ is the heat transfer coefficient, $A$ is the cross-sectional area, and $\partial T / \partial x$ is the temperature gradient. Clearly, choosing material with minimal heat transfer coefficient and minimizing the cross-sectional area are essential to reduce the conductive heat load. Stainless steel was chosen to construct the bore tube because, in addition to having low thermal conductivity, it is also structurally strong, which allows the use of thin wall tube without the risk of implosion. The ion transfer hexapoles were made out of titanium tubes instead of rods, to reduce the cross-sectional area. Finally, all electrical connections down into the $4.2 \mathrm{~K}$ region were made with thin phosphor bronze magnet wires, which have very low heat conductance and reasonable electrical conductance.

The current system has a helium boiloff rate of around $5 \mathrm{~L} /$ day, corresponding to a nearly $0.15 \mathrm{~W}$ 
uncompensated heat load. Although manageable, it is highly desirable if the heat load can be further reduced by $0.15 \mathrm{~W}$ to attain a zero-loss system. This can be achieved by modifying the stainless steel ion guide tube, with its o.d. reduced to about $2.5 \mathrm{~cm}$ (from $7.6 \mathrm{~cm}$ ), just wide enough to house the ion guide, and its wall thickness reduced to $0.5 \mathrm{~mm}$ (from $0.9 \mathrm{~mm}$ ), thick enough to resist crumpling even to $30 \mathrm{psig}$. This should provide a nearly sixfold reduction in heat conduction (from 0.23 to $0.04 \mathrm{~W}$ ), more than enough to eliminate the residual heat load.

Further improvements will involve installation of the low-temperature preamplifier [24] inside the vacuum, near the ICR cell, and chilled to $4.2 \mathrm{~K}$. The present preamplifier is connected to the detect plates by an approximately $2 \mathrm{~m}$ long phosphor bronze wire and an approximately $30 \mathrm{~cm}$ coaxial cable, adding an extra capacitance of about $50 \mathrm{pF}$ at each input of the amplifier, severely attenuating the ICR signal. Moreover, the instrumentation amplifier currently used is robust, but not optimal in terms of signal/noise characteristics. It has been shown that the custom-built silicon JFETbased room-temperature preamplifier yields $>25$-fold lower noise than the commercial in-vacuum preamp, which is also tenfold better than this simple instrumentation amp [25]. The GaAs MESFET-based cryogenic preamplifier has been tested and functions well at $4.2 \mathrm{~K}$, with similar performance enhancement. Keeping the preamplifier inside the vacuum, shielded by the metal walls, will also significantly reduce RF interference.

Finally, the present system cryopumps using a simple aluminum container filled with activated charcoal placed just below the ICR cell. A higher surface area cryo-array is currently being designed, which should provide better pumping speed and base pressure once installed.

\section{Conclusions}

A cryogenic FTMS has been constructed with a $15 \mathrm{~T}$ magnet, which offers several advantages over the conventional warm bore design, including the field strength, base pressure, and preamplifier noise. The helium boiloff rate is currently at a manageable 5 L/day, with modifications under way to reduce the heat transfer further to make it a zero-loss system. First signal has been achieved on this instrument by detecting the LDI $\mathrm{C}_{60}^{+}$ions at $7 \mathrm{~T}$, and later at $14 \mathrm{~T}$. Although not fully optimized, this signal shows the proof of concept for cryo-FTMS. Replacing the commercial room-temperature instrumentation amplifier with the low-temperature preamplifier in proximity to the ICR cell is expected to significantly improve the signal/noise characteristics.

\section{Acknowledgments}

The authors gratefully acknowledge the financial support from the National Heart, Lung, and Blood Institute (Grant NIH/NHLBI NO1HV-28178), the National Center for Research Resources (Grant NIH/NCRR P41RR-10888), and MDS Sciex. The authors also thank Drs. Steve Beu, Gerald Gabrielse, Ron M. A. Heeren, Chris Hendrickson, Ronald Knepper, and Lutz Schweikhard for valuable comments;
Adam Berryhill and Mike Coffey for the magnet design and operation; and personnel from Boston University Scientific Instruments Facility for building critical components for the instrument.

\section{References}

1. Comisarow, M. B.; Marshall, A. G. Fourier Transform Ion Cyclotron Resonance Spectroscopy. Chem. Phys. Lett. 1974, 25, 282-283.

2. Easterling, M. L.; Mize, T. H.; Amster, I. J. Routine Part-per-Million Mass Accuracy for High-Mass Ions: Space-Charge Effects in MALDI FT-ICR. Anal. Chem. 1999, 71, 624-632.

3. Muddiman, D. C.; Oberg, A. L. Statistical Evaluation of Internal and External Mass Calibration Laws Utilized in Fourier Transform Ion Cyclotron Resonance Mass Spectrometry. Anal. Chem. 2005, 77, 2406-2414.

4. Zhang, L. K.; Rempel, D.; Pramanik, B. N.; Gross, M. L. Accurate Mass Measurements by Fourier Transform Mass Spectrometry. Mass Spectrom. Rev. 2005, 24, 286-309.

5. Kim, S.; Rodgers, R. P.; Marshall, A. G. Truly "Exact" Mass: Elemental Composition Can Be Determined Uniquely from Molecular Mass Measurement at Similar to $0.1 \mathrm{mDa}$ Accuracy for Molecules up to Similar to 500 Da. Int. J. Mass Spectrom. 2006, 251, 260-265.

6. Shi, S. D. H.; Hendrickson, C. L.; Marshall, A. G. Counting Individual Sulfur Atoms in a Protein by Ultrahigh-Resolution Fourier Transform Ion Cyclotron Resonance Mass Spectrometry-Experimental Resolution of Isotopic Fine Structure in Proteins. Proc. Natl. Acad. Sci. U.S.A. 1998, 95, 11532-11537.

7. Kelleher, N. L.; Lin, H. Y.; Valaskovic, G. A.; Aaserud, D. J.; Fridriksson, E. K.; McLafferty, F. W. Top down versus Bottom up Protein Characterization by Tandem High-Resolution Mass Spectrometry. J. Am. Chem. Soc. 1999, 121, 806-812.

8. Bogdanov, B.; Smith, R. D. Proteomics by FTICR Mass Spectrometry: Top down and Bottom up. Mass Spectrom. Rev. 2005, 24, 168-200.

9. Frank, A. M.; Savitski, M. M.; Nielsen, M. L.; Zubarev, R. A.; Pevzner, P. A. De Novo Peptide Sequencing and Identification with Precision Mass Spectrometry. J. Proteome Res. 2007, 6, 114-123.

10. Marshall, A. G.; Rodgers, R. P. Petroleomics: The Next Grand Challenge for Chemical Analysis. Acc. Chem. Res. 2004, 37, 53-59.

11. Marshall, A. G.; Hendrickson, C. L.; Jackson, G. S. Fourier Transform Ion Cyclotron Resonance Mass Spectrometry-A Primer. Mass Spectrom. Rev. 1998, 17, 1-35.

12. O'Connor, P. B. Considerations for Design of a Fourier Transform Mass Spectrometer in the $4.2 \mathrm{~K}$ Cold Bore of a Superconducting Magnet. Rapid Commun. Mass Spectrom. 2002, 16, 1160-1167.

13. Winger, B. E.; Hofstadler, S. A.; Bruce, J. E.; Udseth, H. R.; Smith, R. D High-Resolution Accurate Mass Measurements of Biomolecules Using a New Electrospray-Ionization Ion-Cyclotron Resonance Mass-Spectrometer. J. Am. Soc. Mass Spectrom. 1993, 4, 566-577.

14. Gabrielse, G.; Fei, X.; Orozco, L. A.; Tjoelker, R. L.; Haas, J.; Kalinowsky, H.; Trainor, T. A.; Kells, W. Thousandfold Improvement in the Measured Antiproton Mass. Phys. Rev. Lett. 1990, 65, 1317-1320.

15. Hillenkamp, F.; Karas, M.; Beavis, R. C.; Chait, B. T. Matrix-Assisted Laser Desorption Ionization Mass-Spectrometry of Biopolymers. Anal. Chem. 1991, 63, A1193-A1202.

16. Beu, S. C.; Laude, D. A. Elimination of Axial Ejection During Excitation with a Capacitively Coupled Open Trapped-Ion Cell for FourierTransform Ion-Cyclotron Resonance Mass-Spectrometry. Anal. Chem. 1992, 64, 177-180.

17. O'Connor, P. B.; Budnik, B. A.; Ivleva, V. B.; Kaur, P.; Moyer, S. C.; Pittman, J. L.; Costello, C. E. A High Pressure Matrix-Assisted Laser Desorption Ion Source for Fourier Transform Mass Spectrometry Designed to Accommodate Large Targets with Diverse Surfaces. J. Am. Soc. Mass Spectrom. 2004, 15, 128-132

18. O'Connor, P. B.; Costello, C. E.; Earle, W. E. A High Voltage RF Oscillator for Driving Multipole Ion Guides. J. Am. Soc. Mass Spectrom. 2002, 13, 1370-1375.

19. Pittman, J. L.; O'Connor, P. B. A Minimum Thickness Gate Valve with Integrated Ion Optics for Mass Spectrometry. J. Am. Soc. Mass Spectrom. 2005, 16, 441-445.

20. Mize, T. H.; Taban, I.; Duursma, M.; Seynen, M.; Konijnenburg, M.; Vijftigschild, A.; Doornik, C. V.; Rooij, G. V.; Heeren, R. M. A. A Modular Data and Control System to Improve Sensitivity, Selectivity, Speed of Analysis, Ease of Use, and Transient Duration in an External Source FTICR-MS. Int. J. Mass Spectrom. 2004, 235, 243-253.

21. O'Connor, P. B.; Costello, C. E. A High Pressure Matrix-Assisted Laser Desorption/Ionization Fourier Transform Mass Spectrometry Ion Source for Thermal Stabilization of Labile Biomolecules. Rapid Commun. Mass Spectrom. 2001, 15, 1862-1868.

22. Comisarow, M. B. Ion Cyclotron Resonance Spectrometry II; SpringerVerlag: New York, 1982.

23. White, G. K.; Meeson, P. J. Experimental Techniques in Low-Temperature Physics; Oxford University Press: Oxford, UK, 2002.

24. Mathur, R.; O'Connor, P. B.; Knepper, R. W. Electronics for the Cryogenic Fourier Transform Mass Spectrometer. Proceedings of the 54th ASMS Conference on Mass Spectrometry and Allied Topics; Seattle, WA, May/June 2006; DVD-ROM.

25. Mathur, R.; Knepper, R. W. O'Connor, P. B. A Low-Noise, Wideband Preamplifier for a Fourier-Transform Ion Cyclotron Resonance Mass Spectrometer. 2007, In press. 